Historic, Archive Document

Do not assume content reflects current scientific knowledge, policies, or practices. 


\section{7) CURRENT PRICE LIST}

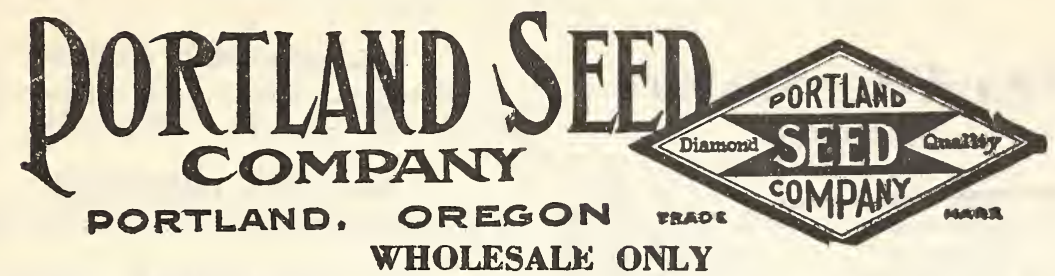

Prices F. O. B. Portland, full bag lots. Subject to change without notice.

PORTLAND, ORE., FEBRUARY 1, 1927

No. 59

We give no warranty, express or implied, as to description, quality, productiveness, or any other matter of any seed Fe send out and will be in no way responsible $\mathrm{f}$ the crop. Purity and germination given for information only and githout guarantee

Prices are per $100 \mathrm{lbs}$. In less ihan $100 \mathrm{lbs}$. reasonable advance will be charged. SEAMLRSS SACKS ARE CHARGRD AT 30c AND ARE NOT RITURNABLE

Law requires Grass and Clover Seeds billed at net weights.

$100 \mathrm{lbs}$.

Alfalfa, Fancy, $99 \%$...........\$20.00

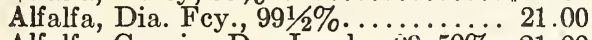

Alfalfa, Genuine Dry Land...99.50\%. 21.00 Alfalfa, Grim, Certified.................... 36

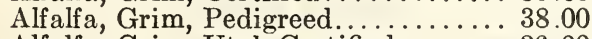
Alfalfa, Grim, Utah Certified........ 36.00 Alfalfa, Grim, Idaho Certified . . . . . . 38.00 Clover, Alsike Ch............... 31.00 Clover, Alsike Fcy.............. 33.00 Clover, Alsike Dia. Fcy............. 34.00 Clover, Alsike \& White Mix......... 30.00

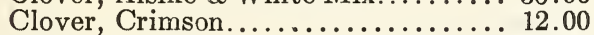
Clover, Red Ch................. 35.00

\section{Grasses}

\section{Blue Grass-}

Kentucky Fcy., 19-lb. seed........\$29.00

Kentucky Ex. Fancy, 21-lb. seed. .. 30.00 Blue Grass, Canadian .............. 38.00 Bromus Inermus...................... 18.00

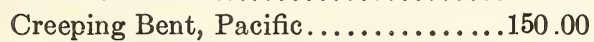

Fesque, Chewing.................... 35.00

Fescue, Hard...................... 30.00

Fescue, Red or Creeping............... 30.00

Fescue, Sheep..................... 30.00

Lawn Grass, Fine Mixed.......... 30.00

Lawn Grass, Diamond Fancy............... 400

Meadow Fescue.................. 22.00

Millet, Golden................... 8.00

Millet, Jap....................... 8.50

Millet, Siberian.................... 8.00

Millet, Ea. Fortune................. 7.00

Millet, Broom Corn or Hog.......... 8.00

Orchard Grass, Fancy........... 18.50

Pasture or Burn Mixture...... No.14.. 10.00

Pasture or Burn Mixture...... No.15.. 11.00

Pasture Mixture, Lowland ...No.16.. 15.00 Upland....No.17.. 15.00

Red Top, Fancy Hulled........... 35.00

Red Top, Dia. Fancy Hulled...........

Rye Grass, West................... 6.50

Rye Grass, English.................... 10.00

Rye Grass, English Imported........ 12.00

Rye Grass, Italian.................. 7.50

Rye Grass, Italian Imp................ 11.00

Sudan Grass................... 10.00

Tall Meadow Oat Grass............ 22.00

Timothy, Choice...........98.5\% . 8.25

Timothy, Fancy..............99.\%. 8.75

Timothy, Dia. Fancy, ......99.5\% . 9.00
100 lbe

Clover, Red Fcy................ 35.50

Clover, Red. Diamond Fancy.......... 36.00

Clover, Red Imported.................3400

Clover, Mammoth................. 37.00

Clover, Sweet Hubam............ 42.00

Clover, Sweet Wht. Blossom, Fcy.... 16.00

Clover, Swt. Yel. Blossom, Biennial. . 17.00

Clover, Swt. Yel. Blossom, Annual... 10.00

Clover, White for Pasture.......... 32.00

Clover, White, Choice.............. 35.00

Clover, White, Fancy................ 37.50

Clover, White Dia. Fancy......... 40.00

Clover, White Ladino ...............100.00

\section{Seed Grains}

Barley, Common.........\$2.50 \$48.00

Barley, Hanchen.............. $3.00 \quad 58.00$

Barley, Success Beardless.... $3.25 \quad 63.00$

Barley, White Hulless........ $3.75 \quad 73.00$

Barley, New Trebi.......... $3.00 \quad 58.00$

Buckwheet, Jap............ 5.00

Buckwheat, Silver Hull....... 5.00

Cane, Early Amber......... 7.50

Cheat................... 2.35

Flax.................. 8.00

Kaffir Corn.............. 6.50

Milo Maize.............. 7.00

Oats, Climax............... 3.10

Oats, Senator............ 3.10

Oats, Eclipse................ 3.10

Oats, Gray.................. 2.50

Oats, Red................... 2.50

Oats, White Seed or Side.... 2.50

Oats, Swedish Select........ 3.00

Oats, Hulless............. 5.75

Tangier Peas....................

Field Peas, White or Blue............

Rape, Dwf. Essex.......... 8.00

Rye, Fall Seed, Recleaned... 2.50

Rye, Recleaned Spring....... 3.50

Rye, Rosen............... 3.00

Vetch and Oats, $50-50 \ldots \ldots . .6 .00$

Vetches, No. 1............. 7.00

Vetch, White Spring..........

Vetches, Purple............. $\ddot{9} .00$

Vetches, Hungarian........... 7.00

Vetches, Sand or Hairy....... 15.00

Wheat, Bluestem........... 3.50

Wheat, Marquis............ 3.50

Wheat, New Federation..... 3.50

Wheat, Club.............. 3.50

45.00

.....

60.00

60.00

60.00

48.00

48.00

48.00

58.00

113.00

12.00

5.00

48.00

68.00

60.00

......

......

......

.......

68.00

68.00

68.00

68.00 


\section{Cream Separators CHAMPION}

No. 3 Separator Stands. .Less 25\% 10.00 No. 11/2 Separator .........Less 25\% 30.00 No. 3 Separator .........Less $25 \% 40.00$ No. 25 Separator .........Less 25\% 50.00 No. 300BB Separators......Less $25 \% 77.50$ No. 500BB Separators......Les's 25\% 85.00 No. 700BB Separators......Less 25\% 90.00 No. 900BB Separators...... Less 25\% 95.00 No. 900 BB Elec. Sep. Less Motor 25\% 125.00

FERTILIZERS-For all purposes. High Grade - no Filler 100 lbs. Ton

Vegetable Fertilizer........\$2.35 \$45.00

Dia. Lawn................ $2.75 \quad 52.00$

Fruit and Onion........... $2.60 \quad 50.00$

Potash, Muriate.......... $2.40 \quad 46.00$

Nitrate of Soda............ $3.25 \quad 61.00$

Sulphate of Ammonia....... $4.25 \quad 81.00$

Superphosphate........... $1.55 \quad 29.00$

Sheep Guano............... $1.00 \quad 18.00$

Fish Guano.............. $3.50 \quad 68.00$

Bonemeal................ 2.45 45.00

Tankage ................ 2.60 50.00

H. O. Lime............. $1.00 \quad 19.00$

Dia. Plant Food $1 \mathrm{lb}$. carton, doz.. $\quad 3.00$

Dia. Plant Food 1/2 lb. carton, doz. $\quad 2.00$

\section{BUCKEYE INCUBATORS Whsle.}

No. 16. Style E, $110 \mathrm{Egg}$, each..... \$11.00

No. 1. Standard, 110 Egg, each..... 24.00

No. 2. Standard, 175 Egg, each...... 26.00

No. 3. Standard, 250 Egg, each...... 30.00

No. 5. Standard, 600 Egg, each..... 75.00

\section{QUEEN INCUBATORS}

Wt. Size Capacity

75 No. 20, Style K..... 70 Egg

90 No. 21, Style K..... 130 Egg

135 No. 22, Style K..... 220 Egg

80 No. 1 Standard..... 85 Egg

95 No. 2 Standard...... 135 Egg

105 No. 3 Standard..... 180 Egg

145 No. 4 Standard...... 275 Egg

185 No. 5 Standard...... 400 Egg

250 No. 25 Standard. . . . 600 Egg

410 No. 35 Standard..... 800 Egg

525 No. 45 Standard......1000 Egg

750 No. 50 Standard, with Turning

Trays.................. 196.00

COAL BROODERS QUEEN

76 No.0, 42-in. canopy. 400 chicks $\$ 12.60$

81 No. 1, 48-in. canopy. 800 chicks 16.63

108 No. 2, 56-in. canopy .1200 chicks 20.65

120 No. 3, 60-in. canopy.1600 chicks 23.80

\section{BUCKEYE BROODERS}

No. 20. Metal, 60 Chick.......... 8.00

No. 21. Metal, 100 Chick................ 10.00

No. 22. Metal, 150 Chick.......... 14.00

BLUE FLAME OIL BURNING

No. 28. 350 Chick............. 15.00

\section{SEMESAR}

Case lots, less $331-3 \%$ from catologue list ass than, case lots, $25 \%$
OIL BROODERS QUEEN

No. 43-32-in. canopy....300 chicks No. 43-42-in. canopy....600 chicks

$\$ 13.15$

15.60

\section{ELECTRIC BROODERS}

No. B up to 500 chicks........... $\$ 18.85$

\section{CHOLERING}

16-oz. Per doz............... \$ 5.28

Quart, $\quad$ Per doz.................... 8.25

1/2-gal., Per doz................ 16.50

1-gal., Per doz.................. 22.00

5-gal., Each.................... 7.70

10-gal., Each..................... 13.75

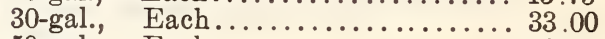

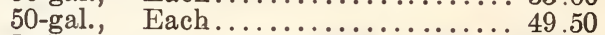

Tablets, 50s., per doz............ 5.28

Tablets, 100 s, per doz........... 7.70

\section{SPRAY PUMPS}

Pomona, Fig. 1100, Pump only....15\%-25.00 Fruit All, Fig. 1188, Pump only...15\%-16.50 Monarch, 2-in. Cyl.............15\%-40.00 Monarch, 21/2-in. Cyl...........15\%-45.00 P. S. Co. Burket Pump............ 3.80 Lever Handle Bucket Pump......... 5.00 Modoc Bucket Pump............. 3.50

Ideal Spray Outfit................ 30.00

\section{LIME AND SULPHUR SOLUTION}

Quart, each ..................\$ 0.25

Gallon " $\ldots \ldots \ldots \ldots \ldots \ldots \ldots \ldots \ldots \ldots \ldots \ldots \ldots \ldots \ldots . .55$

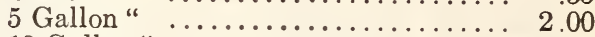

10 Gallon " ........................ 4.00

Barrels" .................. 9.00

\section{DRY LIME AND SULPHUR}

200-lbs............................ Per C

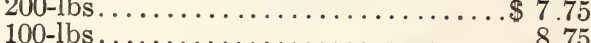

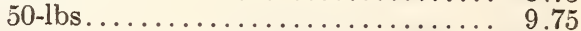

25-lbs............................... 11.00

$10-1$ bs................................... 15.00

5-lbs............................. 17.50

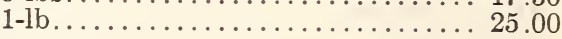

BACILI-KIL B. K. Solution

5 Gals., each $\quad$.............. $\$ 9.40$

1 Gal., each $\quad \ldots \ldots \ldots \ldots \ldots \ldots \ldots \ldots \ldots \ldots \ldots 2.25$

$32 \mathrm{Oz}$, doz. $\quad$................ 10.00

$10 \mathrm{Oz}$, doz. $\quad$.............. 4.80

$4 \mathrm{Oz}$., doz. $\quad$.............. 2.80

Bee Hea!th, gals, each $\ldots \ldots \ldots \ldots .2 .25$

\section{A. WOOD PRESPRVER}

Quarts....(\$2.00 gal.).... 6.00 Doz Gals......(\$1.50 ")..... $18.00 \mathrm{Doz}$. 5 gals.... (\$1.25 “) ..... 6.25 each 55 gal Drum............. 1.00 gal.

\section{BORDEAUX MIXTURE}

$50-1$ bs........................ $\$ 7.50$

25 -lbs........................... 4.00

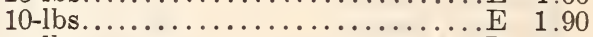

5-lbs........................ 1.15

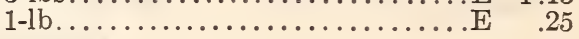




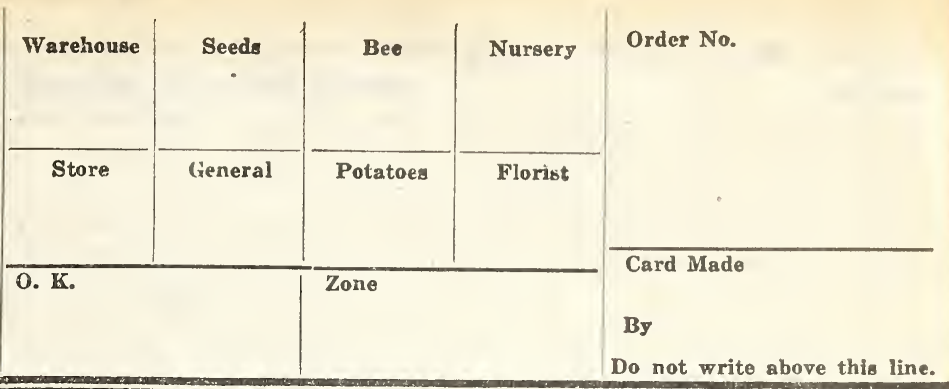

\section{PORTLAMD SEED COMPANY, Porlland, Oregon}

READ INSTRUCTIONS FOR ORDERING ON PAGE 2 OF CATALOG and save all misunderstanding. Write on a separate sheet about any matters not pertaining to this order. This will save delays in filling order.

Name

\section{Write Name and Address Plainly}

\section{Post Office}

R. F. D. No. or Street

Station

County

Be Sure to Give Name of County

State

State if Wanted by Mail, Express or Steamer
Date.

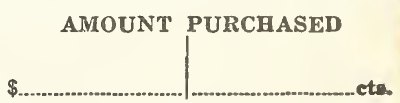

Parcel Post, Express and Freight Station, without an Agent must be prepaid. To cover charges if prepaid add:

$\$$ \$....<smiles>C1CC2CCC12</smiles>

cta.

TOTAL AMOUNT ENCLOSED $\$$ cts.

NOTE.-We use every precaution in procuring fresh, pure and reliable seeds, etc., but we in common with other responsible seed houses, sell our goods subject to the following disclaimer, it being that adopted by the American Seed Trade Association, i. e., We give no warranty, express or implied, as to description, quality, productiveness or any other matter of any seeds, bulbs, nursery stock, roots or plants we sell and will not be in any way responsible for the crop. If the purchaser does not accept the goods on these terms they are to be returned at once.-Portland Seed Co.

\section{Quantity}

Article 


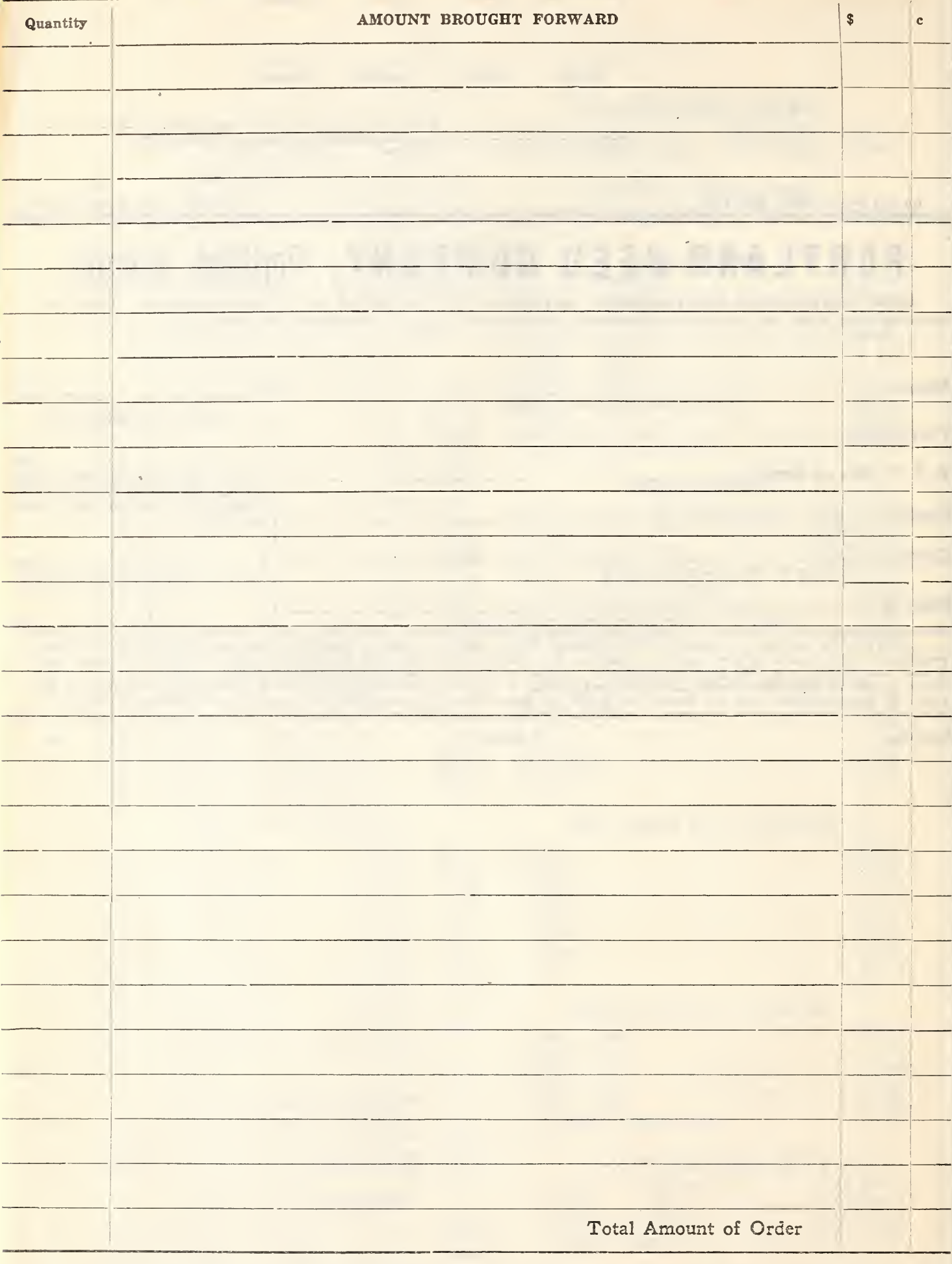

If you have changed your address since you sent us your last order it is important that you fill in the following: MY OLD ADDRESS WAS;

Post Office.

R. F. D. No.

Box No.

State.

Shall we substitute, if for any reason we cannot deliver a variety as ordered. 


\section{A favor to us will mean a gift to you!}

If you will give us the names and addresses of seven or more of your friends who have gardens and might be interested in our catalog, we will include with your order one $10 \mathrm{c}$ packet of seed (your choice) free. This offer is good only until May 1st, 1927.

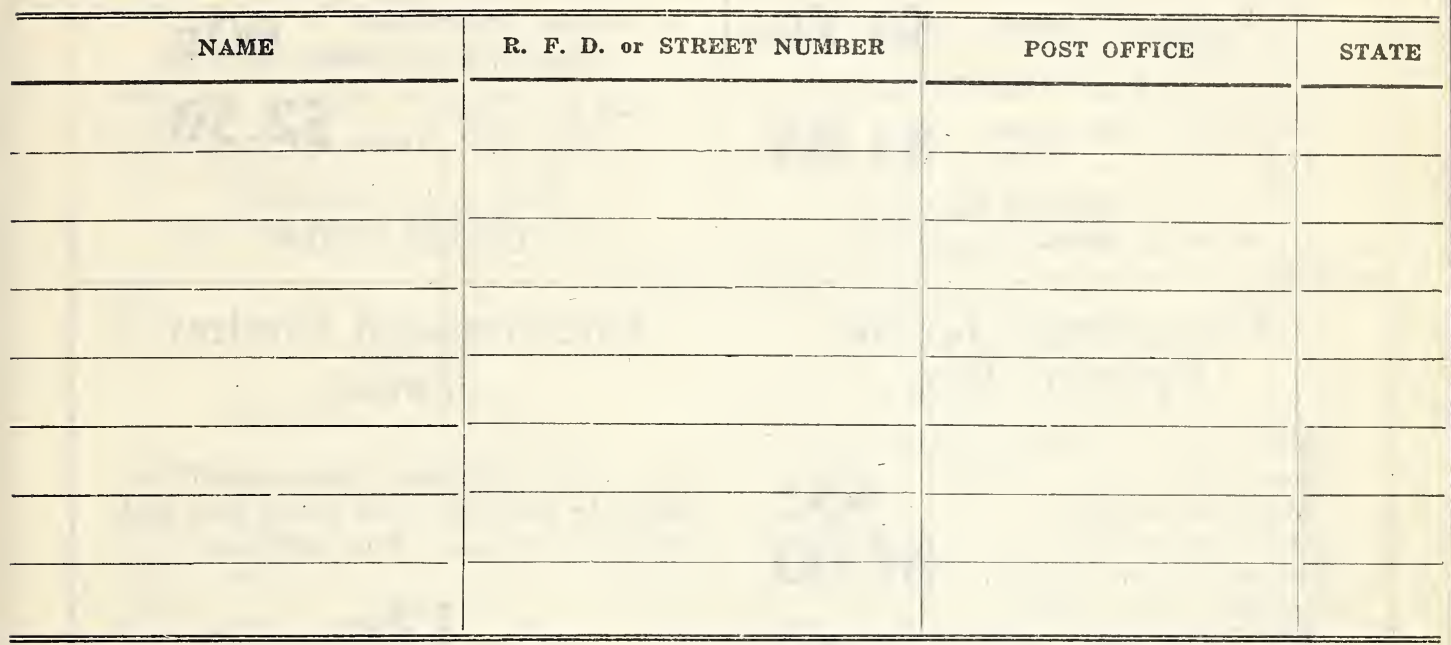

PORTLAND SEED COMPANY: I have filled in the names and addresses of seven or more of my friends. Please include with my order the following $10 \mathrm{c}$ packet of seed FREE.

Name of seed wanted.

\section{Two tools you will want for garden and lawn}

\section{A GARDEN SHOVEL}

A Woman's Size

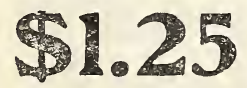

Postage Extra

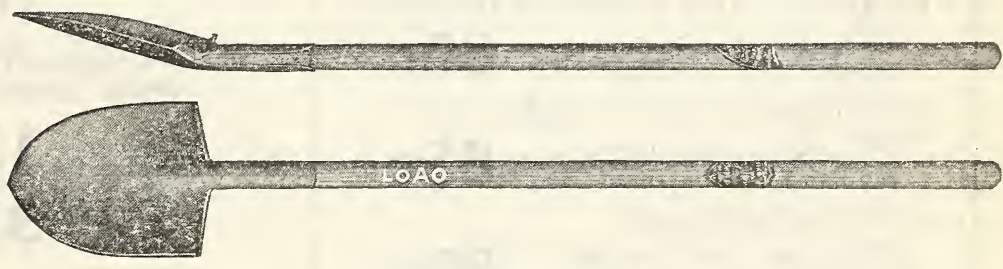

Here is just the right size and shape of long handled, pointed spade for a woman's or older child's use in the garden. Not a plaything but strongly made of the best quality steel, with straight grained hardwood handle. Regular value $\$ 1.65$; special to our customers at $\$ \mathbf{1 . 2 5}$ for a Spring special-NEW- just received.

\section{Dand}

Pulls the root of the dandelion without tearing up lawn; has long handle, polished steel blade, very simple. TRY IT. Mail wt. 2 lbs. Regular 75c value- 


\section{More}

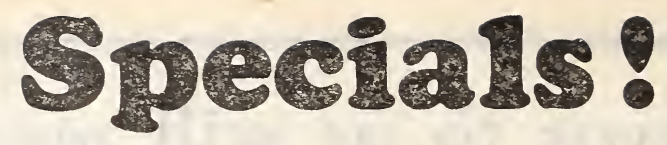

\section{Two Books for Almost the Price of One}

THE AMERCAN APPLE ORCHARD

By Waugh. 214 pages,

cloth bound

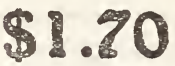

PRINCIPALS OF FRUIT GROWING

By Bailey. 516 pages,

cloth bound

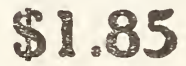

Both for 92.00 Postpaid

Supply Limited-Order Early

\section{Three Books for the} Price of Two

CABBAGE, CAULIFLOWER,

93 pages, postpaid

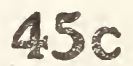

ASPARAGUS-Heximer,

166 pp., clo. bound, postpaid w CELERY CULTURE-

Beattie, 150 pages, cloth

bound, postpaid

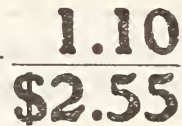

VALUE $\$ 2.5$

All three for $\$ \mathbb{1 . 5 5}$ Postpaid

Supply Limited, Order Early
BROCCOLI, Etc.-Gregory.

\section{Lee's Egg Maker}

2 LB. PACKAGE,

Regular 50c Value.... $30 \mathrm{C}$

5 LB. PACKAGE,

Regular $\$ 1.00$ Value 600

25 LB. PAIL,

Reg. \$4.25 Value

$\$ 2.50$

Prices F. O. B. Portland

ORDER EARLY

\section{International Poultry Tonic}

For better digestion and assimilation; helps the birds get more out of their feed, produce more eggs. Full directions.

\section{5c size $\mathrm{C}$ postage $5 \mathrm{c}$ \\ $\$ 1.00$ size $\$ C$ postage $10 c$}

Special for short time-order early as much as you can use

\section{International Gall Heal}

DRAINING FOR PROFIT \& HEALTH 252 pages, cloth bound; regular $\$ 1.60$, postpaid...

$80 c$

FARM GRASSES OF THE U. S. 248 pages, cloth covered; regular $\$ 1.70$, postpaid..........

$85 c$

THE FARMSTEAD,

350 pages, cloth bound; regular $\$ 1.60$ postpaid.

$80 \mathrm{c}$

More Book Specials

FIRST BOOK OF FARMING

259 pages, cloth bound

regular $\$ 1.35$, special.

$85 \mathrm{c}$

FORAGE CROPS OTHER

THAN GRASSES, postpaid

PRACTICAL FORESTRY

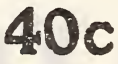

299 pages, cloth bound

$\$ 1.85$-Special, postpaid

$\$ 1.35$

FUNGI \& FUNGICIDES, 228 pages, cloth bound;

$\$ 1.10$-Special
Internationally Known Remedy Special Prices for Short Time

25c size for $2 \mathrm{C}$ postage $5 \mathrm{c}$ 50 c size $33 \mathrm{C}_{\text {postage } 5 \mathrm{c}}$ Order Early

\section{Silver Pine Healing Oil}

An especially fine remedy for cuts, burns, etc. For animals, also very fine for family use.

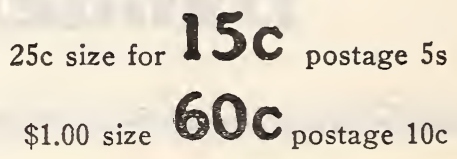
ORDER EARLY 


\section{Poultry and Stock Supplies}

\section{F O B Rail or Boat.}

Poultrymen who look for maximum egg production know it is reached by feeding Diannond Egg Food. This, feed with Diamond Scratch Food brings about and maintains heavy laying and without injury to the layers.

\section{BLATCHFORD'S CALF MEAL}

The perfect milk substitute at one-quarter the cost.

25-lb. sacks, per $100 \ldots \ldots \ldots \ldots \ldots 6.10$

1 ton, 25 -lb. sacks..............117.00

\section{BLATCHFORD CHICK MASH}

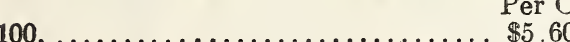

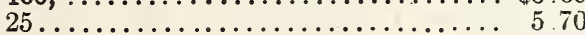

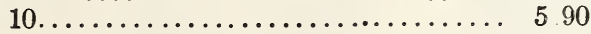

For Worms in Poultry

NOTHING COMPARES

\section{BARNES EMULSION}

16-ox., per doz................ \$4.80

Quart, per doz................. 8.00

Gallon, per doz............... 24.00

5 -gallon, each.................. 8.35

30 -gal. barrel, per gal............ 1.65

50 -gal. barrel, per gal............ 1.60

\section{SEMI SOLID BUTTERMILK Challenge Brand}

5-gallon keg............Per lb

10 -gallon keg..................

$1 / 2$ barrel, 30 gallons............ b

Barrel, 52 gallons................. b

Full information on request.

\section{CHICK AND EGG BOXES}

Chick Boxes, 25 Chicks, doz.......\$2.25

Chick Boxes, 50 Chicks, doz....... 2.75

Chick Boxes, 100 Chicks, doz....... 3.00

Egg Boxes, Kant Krack, 15-Egg, doz. 2.50

Buck Egg Crate, 12-doz. size, complete, each. .................

Bundles, per doz., K.D., no Fillers...

.

Leg Bands, Celluloid, all colors, 100. .

Wire Hens' Nests, each, 20c; doz....

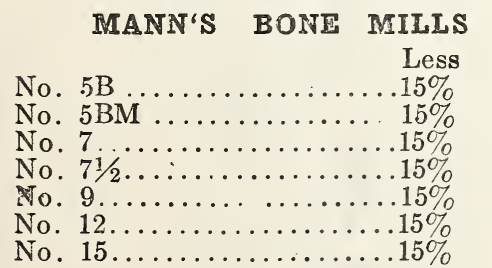

18.50

24.00

27.50

42.00

44.00

80.00

155.00

\section{KOW KARE}

Kow Kare, Small........... \$ .65

Kow Kare, Large........... 1.25

Bag Balm................ $\quad .60$

Garget Cure ................ $\quad .60$

Torse Comf ort.............. $\quad .60$

Horse Comfort............. .30

American Horse Tonic...... . .30

American Horse Tonic...... $\quad .65$

\section{POULTRY AND BIR.D FOODS}

Single Sack, 10c Extra

Diamond Chick Food, per ton.......\$64 00 50-lb. sacks, per ton............66 00 25-lb. sacks, per ton.................6.68 68.00

Diamond Baby Chick Mash.........6. 63.00

Diamond Scratch Food, ton.......... 59.00

Diamond Egg Food, ton........... 5800

Diamond Pigeon Food, ton........... 70.00

Diamond Granulated Bone........... 65.00

Bone Meal Feed....Dia........... 6500

Oyster Shell, Eastern, ton............ 2500

Diamond Grit, X, XX, XXX, ton.... 17.00

Meat Scraps, Swifts, ton........... 85.00

Meat Meal, Swifts................. 85.00

Fish Meal, ton. Dia. Edible........ 95.00

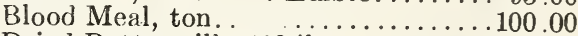

Dried Buttermilk, $100 \mathrm{ibs} . . . \ldots \ldots \ldots . . .12 .00$

Charcoal, ton..................... 6000

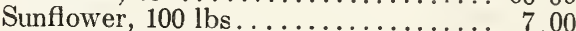

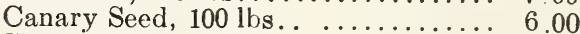

Hemp, 100 lhs ..................... 4.00

Bird Rape, $100 \mathrm{lis}$, Sweet Imported 7.50

Bird Seed, Mixed, Bulk $100 \mathrm{lbs} \ldots . . .6 .75$

“, Mix., cases 60-1 lb ctn. ea. ... 5.50

Roller Bird Seed mix., no Hemp. Swe t

Rape, Doz. $\$ 1.55$, Case 60 lbs.. 7.25

\section{LEE'S GOODS}

Whsle

Retail Doz.

Lice Killer, quart. . . . . . . . \$ $\$ .60 \$ 4.50$

Lice Killer, $1 / 2$ gal . . . . . . . . $90 \quad 7.00$

Lice Killer, 1 gal. ........... $1.50 \quad 12.00$

Lice Killer, 5 gals., each..... $6.00 \quad 4.25$

Egg Maker, 2 lbs........... $\quad 30 \quad 3.00$

Egg Maker, 5 lbs............ $\quad .60 \quad 6.00$

Egg Maker, 25 lb. pails, ea... $\quad 2.50 \quad 2.25$

Egg Maker, 100 lb. Dr., ea.... $9.00 \quad 7.00$

Stock Conditioner, $7 \mathrm{lbs} \ldots \ldots . \quad 1.50 \quad 10.00$

Insect Powder, $1 \mathrm{lb} \ldots \ldots \ldots \ldots . . . .6 \quad 30 \quad 2.25$

Insect Powder, $2 \frac{1}{2} \mathrm{lbs} \ldots \ldots \ldots . \quad .60 \quad 4.50$

Germozone Liquid, 4 oz..... $\quad .40 \quad 3.00$

Germozone Liquid, $12 \mathrm{oz} \ldots \ldots . \quad$....5 5.50

Germozone Liquid, $32 \mathrm{oz} . \ldots . . \quad 1.50 \quad 11.00$

Germozone Liquid, 1 gal., ea. . $4.50 \quad 3.00$

Sheep Dip, Quarts.......... $\quad .75 \quad 6.00$

Sheep Dip, $1 / 2$ gals........... $1.25 \quad 9.00$

Sheep Dip, 1 gals........... $2.00 \quad 15.00$

Sheep Dip, 5 gals. each..... ... 5.50

Egg-O-Hatch............... .50 4.00

Hog Remedy, 71/2 lbs........ 1.50 800

Hog Remedy, pails 25 lbs. ea. . $4.00 \quad 2.00$

Worm Powder, $1 \mathrm{lb} \ldots . . . \ldots \ldots . \quad .35 \quad 2.15$

Garden Insecticide, 1 lb....... $\quad .30 \quad 2.50$

Garden Insecticide, 2 lbs...... .50 4.0n

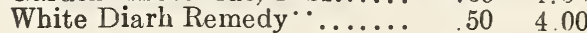


We are now the Northwest Distributers for Conkeys Poultry and Stock Remedies.

Only a partial list below.

\section{POULTRY REMEDIES}

Roup Remedy..........\$0.30

Roup Remedy...........

Roup Remedy..........

Roup Remedy...........

Roup Remedy, 5-lb. cans.

Roup Pills............

Roup Pills.............

Roup Pills

$.604 .80 \mathrm{doz}$.

$1.209 .60 \mathrm{doz}$.

$2.5020 .00 \mathrm{doz}$.

$5.75 \quad 4.00$ each

.302 .40 doz.

Roup Pills, 1000 Pills... $3.00 \quad 2.25$ each

Cholera Remedy....... $\quad .30 \quad 2.40$ doz.

Cholera Remedv........ $\quad .60 \quad 4.80$ doz.

Cholera Remedy....... $1.20 \quad 9.60 \mathrm{doz}$.

Cholera Remedy, 5 lbs... $3.00 \quad 2.25$ each

Poultry Tonic, $11 / 2$ lbs... $\quad .30 \quad 2.40$ doz.

Poultry Tonic, $31 / 4 \mathrm{lbs}$... $\quad .60 \quad 4.80 \mathrm{doz}$.

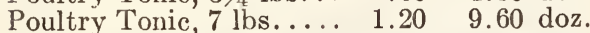

Poultry Tonic, 12-lb. pails $2.0016 .00 \mathrm{doz}$.

Poultry Tonic, 25-lb. pails $3.5032 .00 \mathrm{doz}$.

Poultry Tonic, $50-\mathrm{lb}$. bag. $\quad 6.00 \quad 4.75$ each

Poultry Tonic, 100-lb. bag $11.00 \quad 9.00$ doz.

Lice Powder, 5-oz . . . . . . $25 \quad 2.00 \mathrm{doz}$.

Lice Powder, 15 -oz....... . .45 3.60doz.

Lice Powder, 48-oz...... $\quad .90 \quad 7.20 \mathrm{doz}$

Lice Powder, 12-lb. pail.. $3.00 \quad 2.40$ each

Lice Powder, 25-lb. pail. . $5.25 \quad 4.25$ each

Lice Liquid, 1-qt........ $\quad .60 \quad 4.80$ doz.

Lice Liquid, 2-qt. . . . . . . $\quad .90 \quad 7.20$ doz.

Lice Liquid, 1-gal. . . . . 1.5012 .00 doz.

Lice Liquid, 5-gal........ $7.00 \quad 4.50$ each

Head Lice Ointment, 1-oz. $\quad .15 \quad 1.20$ doz.

Head Lice Ointment, 3-oz. $\quad .30 \quad 2.40$ doz.

White Diarrhea Remedy. $\quad .30 \quad 2.40$ doz.

White Diarr'hea Remedy. $\quad .60 \quad 4.80$ doz.

White Diarrhea Remedy. $1.20 \quad 9.60$ doz.

White Diarrhea, 1200 Tabl $4.00 \quad 2.65$ each

\section{STOCK SPECIALTIES}

Stock Tonic, $1-1 b \ldots \ldots \ldots \$ 0.30 \quad \$ 2.40$ doz.

Stock Tonic, $21 / 4-\mathrm{lb} \ldots \ldots . \quad .60 \quad 4.80 \mathrm{doz}$.

Stock Tonic, 12-lb. pail. . 2.5020 .50 doz.

Stock Tonic, 25-lb. pail. . $4.25 \quad 38.50$ doz.

Stock Tonic, $50-\mathrm{lb}$. bag... $\quad 6.25 \quad 5.00$ each

Stock Tonic, 100-lb. bag.. $11.50 \quad 9.50$ each

\section{NAPTHOLIN}

To keep the bugs away

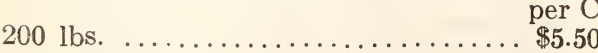

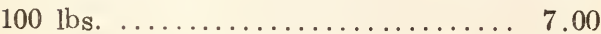

Each

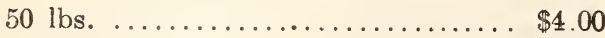

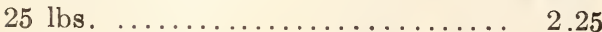

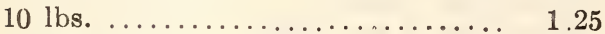

5 lbs. ....................... 0.75

$1 \mathrm{lb} . \ldots \ldots \ldots \ldots \ldots \ldots \ldots \ldots \ldots \ldots \ldots . \ldots .25$

Write for quantity prices
BEE SUPPLIES

\section{WHOLESALE PRICES \\ F.O.B. PORTLAND}

Each

Hives, Fig. 1-8, Pine or Cedar $\$ 3.75$

Hives, Fig. 2-8, Pine or Cedar 3.20

Hives, Fig.10-8, Pine or Cedar 3.65

Hives, Fig.1-10,Pine or Cedar 4.05

Hives, Fig.2-10,Pine or Cedar 3.35

Hives, Fig. 10-10, Pine or Cedar 3.75

Covers, 8-Frame......... . 60

Covers, 10 -Frame............. .65

Bottom Boards, 8-Frme..... $\quad .40$

Bottom Boards, 10-Frme..... . .45

Supers, Fig. $1-8 \ldots \ldots \ldots \ldots \ldots$. 1.20

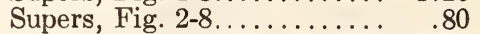

Supers, Fig. 1-10......... 1.55

Supers, Fig. 2-10......... .95

Sections, $41 / 4 \times 13 / 4$, No. $1 \ldots \ldots 14.00 \mathrm{~N}$

Sections, $41 \frac{1}{4} \times 1 \frac{3}{4}$, No. $2 \ldots$... $12.50 \mathrm{M}$

Section Holders, $13 / 4 \ldots \ldots \ldots . \quad .04$

Brood Frame Standard..... $\quad .06$

Brood Frame, Metal Spaced. $\quad .06$

Brood Frame Extracting.... $\quad .06$

Slotted Separators............ .75 C

Veils, No. 1 Silk Tulle..... 1.40

Veils, No. 2 Silk Tulle Face. 1.25

Veils, No. 3 Cotton Tulle... 1.10

Veils, Globe............. 1.50

Veils, Alexander ............ 1.35

Shipping Cases, $41 / 4 \ldots \ldots \ldots . \quad .50$

Fdn. Fasteners, Parker's.... $\quad .40$

Fdn. Fasteners, Electric.... 1.25

Spur Wire Imbedder....... . .40

Fdn. Med. Brood, .........lb ...75

Fdn. Light Brood, . . . . . . - - . . .80

Fdn. Light Section, .......-lb ...85

Fdn. Ex. Lt. Section, . . . - -lb ..90

$70.00 \mathrm{C}$

$75.00 \mathrm{C}$

$80.00 \mathrm{C}$

$85.00 \mathrm{C}$

Each

Smokers, Clarke.................\$ 1.00

Smokers, Doctor................. 1.40

Smokers, Little Wonder................ .95

Smokers, Jumbo or Engine. . . . . . . . . 1.80

Extractors, Novice, 2-Fr............... 30.00

Extractors, Cowan, 2-Fr. Rev........ 40.00

Extractors, Roots, 4-Fr., Hand...... 85.00

\section{Honey Boards}

No. 11-8-Fr. Unbound............... . .35

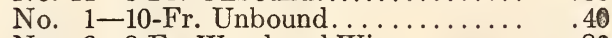

No. $8-8-F r$. Wood and Wire......... .80

No. 7-10-Fr. Wood and Wire....... .90

8-Fr.-7 Wire................ .9.

$10-F r . ~-7$ Wire................... 1.00

Alley Traps, W. \& W., 8-Fr......... $\quad .90$

Alley Traps, W. \& W., $10-$ Fr. . . . . . 1.00

Porter Bee Escapes................. ${ }_{.20}$

Porter Honey House Escape........... . .20

Feeders, Miller's................... $\quad .75$

Feeders Division Board.............. $\quad .40$

Feeders, Boardman's, no jar.......... $\quad .30$

Feeders, Alexander................. .65 